classroom. The use of gamification is possible without information technologies, but the use of computer games or online components will enrich educational games and benefit the training of specialists. The difficulties of introducing gamification into the educational process are determined by the complexity of preparation of educational tasks, the necessity of using the technical means, possible difficulties in the use of them in the classroom and computer literacy of teachers.

In Ukraine, gamification is not widespread, however, taking into consideration a number of advantages over traditional approaches, it can be gradually introduced into the educational process in order to modernize the teaching of foreign languages in the higher educational institutions of Ukraine. Examples of the usage of gaming technology in education are online services and educational platforms. Duolingo is a service for self-study of a foreign language, which at the same time provides a service for its use in educational institutions. Edmodo and Kahoot are game-based learning platforms that can be used in foreign language classes.

Gamification helps to teach effectively, motivates cooperation and interaction between participants, but it should be remembered that the use of game technology in institutions is not entertainment or a substitute for the teacher. Gamification of the educational process, according to the author, in some cases is more effective in teaching modern students comparing to traditional methods and successfully complements them. Taking into account the above, it is important to introduce moderate gamification in the educational process in Ukraine, considering all the advantages and difficulties of this phenomenon.

Key words: gamification, gaming technologies, game, game activity, gamification of education.

DOI: https://doi.org/10.32782/2410-0927-2020-12-7

УДК 821.112.2:339.5

Olena Halytska

\title{
VON GASTARBEITERLITERATUR ZUR TRANSNATIONALEN LITERATUR? PROBLEMATISIERUNGEN UND FORSCHUNGSFRAGEN
}

Innerhalb der Literaturwissenschaft herrscht eine gewisse Begriffsunsicherheit: Es gibt viele verschiedene Begriffe, welche zum Teil bedeutungsgleich verwendet werden, zum Teil allerdings auch mehr oder weniger strikt voneinander unterschieden werden (transnationale Literatur, Chamisso-Literatur, interkulturelle Literatur, diasporische Literatur, Literatur der Betroffenheit, Minderheitsliteratur, Literatur mit dem Motiv der Migration, Literatur der Migration, Fremdliteratur, Literatur der Fremde, Literatur in der Fremde, Ausländerliteratur, Literatur ohne festen Wohnsitz, Gast-, Immigranten-, Emigrations-, Migranten-, Migrationsliteratur, deutsche Gastliteratur, deutsche Literatur von außen, Exilliteratur, die Literatur der (Arbeits-)Migranten. In der Gegenwart wird viel über die Neudefinierungen von Migrantenliteratur "interkulturelle Literatur" und "transnationale Literatur" debattiert und gestritten. Neuere Befunde der Migrationsforschung lassen erkennen, dass soziale und kulturelle Motive vor geographischen und ökonomischen Wanderungsmotiven gegenwärtig dominieren. Zwischen Transmigration und transnationaler Literatur, die in das 21. Jahrhundert hinüberleitet, besteht ein zwingender Zusammenhang. Wichtige Kriterien der transnationalen Literatur sind: die mehrsprachige Erfahrung; die mehrkulturelle Erfahrung in der Minderheitensituation; neue transnationale Erinnerungskulturen, in denen divergente Bedeutungen aufeinandertreffen, zentrale Ereignisse der Geschichte und Persönlichkeiten und Prozesse neu bewertet und tradierte Narrative befragt und transformiert werden. Diese Literatur zeichnet sich durch einen transnationalen respektive transkulturellen Charakter aus. Phänomene der Transnationalität gibt es in der Literatur auf drei Ebenen: Produktion, Thematik und Darstellung, Rezeptio. Dabei ist zu unterscheiden: nach Autoren, die bereits in ihrer Heimat und in ihrer Muttersprache als Autoren waren (Herta Müller (Rumunien), Maynat Kurbatowa (Tschetschenien), Marjana Gaponenko (Ukraine)); nach Autoren, die erst durch die Erfahrung der Migration zum Schreiben kommen (Abbas Khider (Irak), Katja Petrowskaja (Ukraine), Liu Dejun (China)); nach Autoren, die noch in jungem Alter ihre Heimat verlassen mussten und im deutschsprachigen Raum aufgewachsen sind (Z Şenocak, J F A Oliver, Z Çirak, S Özdogan, Feridun Zaimoglu, Saša Stanišić); nach Autoren mit Migrationshintergrund, deren Elternteile beide (oder ein Elternteil) im Ausland geboren wurden, sie sind von Kind an bi- oder trilingual (Shida Bazyar, Sherko Fatah).

Schlüsselwörter: transnationale Literatur, transnationale Erinnerungskulturen, Transmigrant.

Einleitung und Fragestellung. Der Begriff der transnationalen Literatur ist sehr facettenreich und wird stark diskutiert. Innerhalb der Literaturwissenschaft herrscht eine gewisse Begriffsunsicherheit und es deshalb wichtig ist, einen Überblick über unterschiedliche Auffassungen von transnationalen Literatur zu geben.

Das Ziel dieser Arbeit ist, den wissenschaftlichen Diskurs zur transnationalen Literatur in seinen wichtigsten Punkten wiederzugeben.

(C) Halytska O., 2020 
In der Theorie gibt es viele verschiedene Begriffe der transnationalen Literatur, welche zum Teil bedeutungsgleich verwendet werden, zum Teil allerdings auch mehr oder weniger strikt voneinander unterschieden werden. werden.

Die für diese Arbeit bedeutendsten Begriffe sollen im Folgenden zusammenfassend dargestellt

Der erste Terminus "Gastarbeiterliteratur" entwickelte sich bereits in den 70er- und 80er-Jahren des 20. Jahrhunderts, welcher später als Basis für die Begriffsbildung anderer Wissenschaftler dienen sollte (vgl. [18]). Es geht hier viel mehr um die Literatur, die von ausländischen Schriftstellern in Deutschland, Österreich und in der Schweiz geschaffen wurde. Der Begriff der Gastarbeiterliteratur lehnt sich an die Tatsache an, dass man um 1955 der Bedarf an Arbeitskräften in der Industrie und Bauwirtschaft in den deutschsprachigen Ländern stieg und diese als Gastarbeiter (statt Fremdarbeiter) bezeichnete.

Dieser erste Begriff der Gastarbeiterliteratur wurde in den folgenden Jahren von vielen Seiten kritisiert. Vielen Kritikern war dieser Begriff unzutreffend. So betont zum Beispiel der 1940 in Izmir geborene Schriftsteller Yüksel Pazarkaya, dass er kein Gastarbeiterautor sei, und seine Texte keine Gastarbeiterliteratur seien [1, S. 60].

Die Mitglieder des PoLiKunstvereins (1980-1987) Franco Biondie, Rafik Schami, Jusuf Naoum und Suleman Taufiq widersprechen dem oben beschriebenen Begriff "Gastarbeiterliteratur" in einigen wesentlichen Punkten und verwenden den Begriff "Literatur der Betroffenheit", die "unterdrückte Literatur" bedeutet [3, S. 124-136].

Der Linguist Harald Weinrich konstatiert 1986, dass es die Zeit gekommen ist, "den viel differenzierteren Charakter dieser Literatur wahrzunehmen" [1, S. 98]. Des Weiteren ist zu erwähnen, dass Sigrid Weigel in ihrer Publikation "Literatur der Fremde - Literatur in der Fremde" [21], "den interkulturellen Vernetzungen von Wahrnehmung des Fremden und der Entstehung von "Texten kultureller Minoritäten" nachzuspüren" versucht [18, S. 105]. Der auf Deutsch schreibende Türke Feridun Zaimoglu betont, dass er ein deutscher Autor sei [18, S. 107].

Ergebnisse. Wenn man wissenschaftliche Literatur zum Thema "transnationale Literatur" studiert, so kann man eine bestimmte terminologische Vielfalt konstatiert werden: Chamisso-Literatur [7], interkulturelle Literatur [19], diasporische Literatur, Literatur der Betroffenheit [3], Minderheitsliteratur, Literatur mit dem Motiv der Migration, Literatur der Migration [20], Fremdliteratur, Literatur der Fremde [21], Literatur in der Fremde [21], Ausländerliteratur [1], Literatur ohne festen Wohnsitz, Gast-, Immigranten-, Emigrations-, Migranten-, Migrationsliteratur [4; 8; 16; 17], deutsche Gastliteratur, deutsche Literatur von außen, Exilliteratur, die Literatur der (Arbeits-)Migranten (vgl. [2, S. 7]). Aber es wird keine strenge terminologische Unterscheidung zwischen diesen Bezeichnungen eingehalten.

Migrationsliteratur "als eine Literatur in Bewegung" [11, S. 23] ist einer der neueren Begriffe. "Unter diesem Begriff versammeln sich Bücher von Autoren, die ihr Geburtsland verlassen haben und ihre Lebenserfahrung im Dazwischen in den Mittelpunkt ihres Schreibens stellen." [8, S. 11].

Bereits der Titel des Handbuches von Walter Schnitz "Literatur der Migration in den deutschsprachigen Ländern seit 1945" [20] verrät dem Leser viel über dessen Inhalt. Es geht um die Entwicklung der Literatur der Migration, Konzepte, Phasen, Kontexte, Autorinnen und Autoren und ihre Werke.

Diskursgeschichtlich ist die Verwendung des Terminus "Migrationsliteratur" im Laufe der vergangenen knapp 60 Jahren Veränderungen unterworfen gewesen. Denn Autoren und Autorinnen mit Migrationsgeschichte müssen heute nicht mit ihren individuellen und sozialpolitischen Problemen kämpfen, sie kämpfen um ein neues literarisches Leben und haben die Möglichkeit, jeden existierenden Literaturpreis zu gewinnen und nicht nur Adalbert-von-Chamisso-Preis (1984-2017), der für Deutsch schreibende Autoren und Autorinnen nichtdeutscher Herkunft und Muttersprache eingerichtet wurde.

Wie bereits erwähnt, hatte man auch noch eine Alternativbezeichnung "Chamisso-Literatur" im Blick. Im Zentrum der Untersuchung von A. Catone sind mehr- und gemischtsprachige literarische Texte von Yoko Tawada, Ilma Rakusa, Vladimir Vertlib, mit denen kulturelle Grenzen überwunden werden können [7]. 
Deutlich ist eine interkulturelle Vielfalt. "Besonders der Sprach- und Kulturwechsel durch Migration hat diese Vielfalt bewirkt - oft als schmerzhafte Erfahrung." [17, S. VII].

In der Gegenwart wird viel über die Neudefinierung von Migrantenliteratur "interkulturelle Literatur" debattiert und gestritten. Für solche laufende Debatte sind literatursoziologische Theorien wichtig: Sievers' Sammlung "Grenzüberschreitungen. Ein literatursoziologischer Blick auf die lange Geschichte von Literatur und Migration" [19] leistet einen rechtzeitigen Beitrag zu diesem Bereich. Dabei sollten Texte von immigrierten SchriftstellerInnen als Avantgarde verstanden werden, weil sie "diese neue Welt imaginiert" [19, S. 10]. Im Vordergrund der Forschung stehen in Österreich lebenden zeitgenössischen Autorinnen und Autoren mit Migrationshintergrund (Ilir Ferra, Stanislav Struhars, Tanja Maljartschuk, Elias Canetti, Milo Dor, György Sebestyén, Seher Çahir), die bisher fast überhaupt nicht erforscht wurden.

Der deutsche Literaturwissenschaftler Norbert Mecklenburg war einer der Ersten im deutschen Raum, der eine Definition der interkulturellen Literaturwissenschaft liefert: "Interkulturelle Literaturwissenschaft gab und gibt es überall dort, wo Literaturwissenschaftler bei ihrer Arbeit Kulturunterschiede bedenken und über Kulturgrenzen hinausdenken" (zit. nach [13, S. 9]).

Neuere Befunde der Migrationsforschung lassen erkennen, dass soziale und kulturelle Motive vor geographischen und ökonomischen Wanderungsmotiven gegenwärtig dominieren. Migranten heute erscheinen als multilokale oder pluri-lokale Grenzgänger, die sich "in transnationalen Sozialräumen” (s. $[15$, S. 39-40]) befinden.

Damit keine Missverständnisse entstehen, sei hinzugefügt, dass man vier Typen internationaler Migranten unterscheidet [15, S. 39-40]:

1) Emigranten bzw. Immigranten (sie "unterhalten zwar noch Kontakte zu ihren Herkunftsländern, richten sich jedoch auf Dauer in den Ankunftsländern ein und integrieren sich schrittweise als Eingewanderte");

2) Rückkehr-Migranten / Remigranten (sie wechseln ihren Wohnort saisonal oder für einige Jahre zum Zwecke des Gelderwerbs und kehren dann in ihr Herkunftsland zurück); bestimmt);

3) Diaspora-Migranten (ihre grenzüberschreitende Wanderung ist z. B. religiös oder politisch

4) Transmigranten / transnationale Migranten (das Verhältnis zwischen Herkunfts- und Ankunftsregion ist "durch die Herausbildung von auf Dauer angelegten transnationalen sozialen Räumen gestaltet”).

Zwischen Transmigration und interkultureller Literatur besteht ein zwingender Zusammenhang in der globalisierten Welt, wo man global denkt und Differenzen akzeptiert, aber man hat einen gemeinsamen Welthorizont. Der Pfad, der aus der Beengung der nationalen Literatur hinauszuführen verspricht, ist Mehrsprachigkeit ("Weltläufigkeit im Literarischen") [4, S. 79]. Die Forschung zu im Kontext von Transmigration entstandenen Literatur ist transnationale Literatur, die in das 21. Jahrhundert hinüberleitet.

Die Literaturwissenschaft hat bei der Erforschung der transnationalen Literatur in den letzten zwei Dekaden gute Fortschritte gemacht, angefangen von Franciszek Grucza (2012) bis hin zu Eva Hausbacher (2009, 2019), Esther Kilchmann (2019) um nur einige zu nennen. Durch diese Forschung hat sich die Ansicht, viele der Texte von SchriftstellerInnen anderer sprachlicher und kultureller Herkunft, die in deutscher Sprache geschrieben wurden, spiegeln "die andere kulturelle Erfahrung der Autoren in ihrem Herkunftsland, ihre Identitätssuche und die Auseinandersetzung mit der Situation als Fremder in Deutschland, Österreich und der Schweiz sowie ihre individuellen und sozialpolitischen Probleme und Erfahrungen" wider, gewandelt zu dem Verständnis, dass diese Autoren "nicht auf die Ausländerthematik allein festgelegt werden können" [6, S. 498]. Wichtigere Kriterien sind [6, S. 498]:

- die mehrsprachige Erfahrung (z. B. Katja Petrowskajas Texte sind Delirium zwischen den Sprachen. Einige sind im Gewühl zwischen den Sprachen entstanden. Der Artikel von Holger Heiman über Katja heißt "Die deutsche Sprache kam einer Befreiung gleich" (Welt, 08.07.2013));

- die mehrkulturelle Erfahrung in der Minderheitensituation;

- neue transnationale Erinnerungskulturen, "in denen divergente Deutungen aufeinandertreffen, Ereignisse neu bewertet und tradierte Narrative befragt und transformiert werden" [4, S. 156]. 
Anja Tippner weist meines Erachtens zu Recht darauf hin, dass bei der Bewegung über Grenzen hinweg und dem Transfer von einer nationalen Erinnerungskultur in eine andere "neue, transnationale Erinnerungskulturen, in denen divergente Deutungen aufeinandertreffen, Ereignisse neu bewertet und tradierte Narrative befragt und transformiert werden," entstehen. [6, S. 156]. Folgerichtig wäre, dass historische Ereignisse, Persönlichkeiten und Prozesse ganz unterschiedliche Bedeutungen in verschiedenen nationalen Erinnerungskulturen haben. Beispielsweise hat der 47-jährige deutschirakische Schriftsteller Abbas Khider andere Erinnerungsnarrative an die Grausamkeiten des irakischen Regimes (s. sein Roman "Palast der Miserablen") im Gepäck als Menschen, die etwa aus Deutschland oder der Ukraine kommen.

Die 1970 in Kyjyw geborene Schriftstellerin, eine Ukrainerin mit jüdischen Wurzeln, Katja Petrowskaja versammelt in ihrem Debütromen "Vielleicht Esther" (2014, Suhrkamp Verlag) ein Tableau von Figuren, das ein ganzes Jahrhundert umfasst. Das Resultat ist ein Mosaik von Erinnerungen.

Tägliche Notizen auf der Facebook-Seite (seit November 2009) des deutsch-syrischen Autors Aboud Saeed werden Gegenstand seines Romans "Der klügste Mensch im Facebook", der 2013 in einem kleinen Berliner E-Book-Verlag "mikrotext" erschien.

Für Rosa Yassin Hassan (Syrien), die seit 2012 in Hamburg lebt, wäre Deutschland immer Exil. Sie hofft auf eine Rückkehr in ihre Heimat.

Phänomene der Transnationalität gibt es in der Literatur auf drei Ebenen [4, S. 384]:

- Produktion

- Thematik und Darstellung

- Rezeptio.

Dabei ist zu unterscheiden (vgl. [6, S. 498]):

- nach Autoren, die "bereits in ihrer Heimat und in ihrer Muttersprache einen Status als Autoren erworben hatten" (O. Filip, A. Skármeta, F. Baykurt, A. Özakin, H. Müller, M. Kurbatowa, M. Gaponenko);

- nach Autoren, die erst durch die Erfahrung der Migration zum Schreiben kommen (F. Biondi, G. Chiellino, SAID, S. Dikman, E. S. Özdamar, K. Kurt, A. Khider, K. Petrowskaja, L. Dejun);

- nach Autoren, die noch in jungem Alter ihre Heimat verlassen mussten und im deutschsprachigen Raum aufgewachsen sind (Z. Şenocak, J. F. A. Oliver, Z. Çirak, S. Özdogan, F. Zaimoglu, R. Knapp, D. A. Franzetti, Z. Bank, S. Stanišić);

- nach Autoren mit Migrationshintergrund, deren Elternteile beide (oder ein Elternteil) im Ausland geboren wurden, sie sind von Kind an bi- oder trilingual (S. Bazyar, S. Fatah).

Terézia Mora ist 1971 in Ungarn geboren. Ihre Familie ist Teil der deutschsprachigen Minderheit, darum wächst sie zweisprachig auf. Ihr Weg beginnt in Sopron (Ungarn) mit dem lyrischen Namen Westtransdanubien. 1990 zieht sie nach Berlin. Seit 1998 ist sie freie Schriftstellerin: Ihre Werke schreibt sie in deutscher Sprache. 2015 wählte die Deutsche Akademie für Sprache und Dichtung (eine Vereinigung von Schriftstellern und Gelehrten, die sich der Pflege, Vertretung und Förderung der deutschen Literatur und Sprache zur Aufgabe gemacht hat) sie zum Mitglied.

Fazit. Diese Literatur zeichnet sich durch einen transnationalen respektive transkulturellen Charakter aus. Die Bücher der transnationalen Autoren sind Schlüssel zu einer Welt, von der wir so wenig wissen.

\section{References}

1. Ackermann, Irmgard, und Harald Weinrich, Hrsg. 1986. Eine nicht nur deutsche Literatur. Zur Standortbestimmung der Ausländerliteratur. München : Piper Verlag.

2. Aktürk, Aysegül. 2007. Interkulturelles Lernen am Beispiel von türkischer Migrantenliteratur im Deutschunterricht. Hamburg : Bedey Media.

3. Biondi, Franco, und Rafik Schami. 1984. "Literatur der Betroffenheit. Bemerkungen zur Gastarbeiterliteratur". In Zu Hause in der Fremde. Ein bundesdeutsches Ausländer-Lesebuch, hrsg. von Christian Schaffernicht, 124 136. Fischerhude.

4. Bischoff, Doerte und Susanne Komfort-Hein, ed. 2019. Handbuch Literatur \& Transnationalität. Berlin, Munich, Boston : De Gruyter.

5. Brezina, Karel. 2003. Funktion und Bedeutung der Migrationsliteratur im deutschsprachigen Raum. München: GRIN Verlag. 
6. Burdorf, Dieter, Christoph Fasbender und Burkhard Moennighoff, Hrsg. 2007. Metzler Lexikon Literatur. Stuttgart, Weimar : J. B. Metzler.

7. Catone, Antonella. 2016. Chamisso-Literatur. Ihr didaktisches Potenzial im universitären DaFLiteraturunterricht in Italien. Marburg : Tectum Verlag.

8. Freudenstein-Arnold, Christiane. 2016. Kindler Kompakt. Deutsche Literatur der Gegenwart. Stuttgart: Metzler.

9. Grucza, Franciszek, Hrsg. 2012. "Vielheit und Einheit der Germanistik weltweit". In Nationale und transnationale Identitäten in der Literatur, Bd. 6. Frankfurt u. a. : Lang.

10. Hamm, Horst. 1988. Fremdgegangen freigeschrieben. Würzburg : Königshausen und Neumann Verlag.

11. Hausbacher, Eva. 2009. Poetik der Migration. Transnationale Schreibweisen in der zeitgenössischen russischen Literatur. Tübingen : StauFFenburg Verlag.

12. Khider, Abbas. 2020. Palast der Miserablen. München : Hanser.

13. Leskovec, Andrea. 2011. Einführung in die interkulturelle Literaturwissenschaft. Darmstadt: WBG.

14. Moslund, Sten Pultz. 2010. Migration literature and hybridity: the different speeds of transcultural changes. Basingstoke : Palgrave Macmillan.

15. Pries, Ludger. 2010. Internationale Migration. Bielefeld : transcript Verlag.

16. Rösch, Heidi. 1992. Migrationsliteratur im interkulturellen Kontext. Eine didaktische Studie zur Literatur von Aras Ören, Aysel Özakin, Franco Biondi und Rafik Schami.

17. Schenk, Klaus, Almut Todorow und Milan Tvrdik, Hrsg. 2004. Migrationsliteratur. Schreibweisen einer interkulturellen Moderne. Tübingen, Basel.

18. Schmidt-Bergmann, Hansgeorg. 2010. Von der "Gastarbeiterliteratur" zu einer "neuen deutschen Literatur". Migration und Integration in der deutschsprachigen Gegenwartsliteratur. http:/www.Bitburger_Gespr 2010_I_Schmidt-Bergmann_99-107.pdf

19. $\overline{\text { Sievers, }}$ Wiebke, Hrsg. 2016. Grenzüberschreitungen. Ein literatursoziologischer Blick auf die lange Geschichte von Literatur und Migration. Wien : Böhlau.

20. Schmitz, Walter. Literatur der Migration in den deutschsprachigen Ländern seit 1945.

21. Weigel, Sigrid. 1992. "Literatur der Fremde - Literatur in der Fremde". In Gegenwartsliteratur seit 1968, hrsg. von Klaus Briegleb und Sigrid Weigel, 182-229. Hanser, München und Weimar.

Галицька Олена. Від літератури гастарбайтерів до транснаціональної літератури: проблематика і дослідницький аспект. Особливої ваги у сучасному німецькомовному просторі набуває транснаціональна література, яка несе припускає транскультурний характер, що реалізується за допомогою багатомовного чи мішаного мовленнєвого інструментарію. В сучасному культурному полілозі не відбувається повного синтезу і злиття, що й зумовлює постійні закономірні зміни в розумінні та тлумаченні поняття транснаціональної літератури. Збереження i репрезентація культурної самобутності в творах транснаціональних авторів Німеччини XXI ст. дає змогу моделювати в країні нову культурну свідомість, яка базується на ідеях мультикультуралізму, етнічного плюралізму, що висуває асиміляцію мігрантів, але не абсолютну, а із збереженням їхньої культурної ідентичності. Транснаціональні автори борються за своє існування в літературному просторі Німеччини.

У статті звернено увагу на наявність значної кількості термінів для позначення поняття транснаціональної літератури і проведено їхнє термінологічне розмежування або уподібнення. На підставі вивчення лексикографічних матеріалів і сучасних лінгвокультурологічних та літературознавчих праць проаналізовано численну термінологію: міжкультурна література, Шаміссо-література, література мігрантів, література гастарбайтерів, література меншості, література іноземців, література імігрантів, література еміграції, література розгубленості тощо. Перші твори транснаціональних авторів було присвячено темі подолання конфліктів, зумовлених упередженим ставленням до гастарбайтерів. На зміну цій тематиці прийшли питання пошуку себе і свого місця в новому транскультурному просторі, пошуку третього простору для нових гібридних транснаціональних ідентичностей в зоні розмитості й інтерференції. Тобто внаслідок пересікання кордонів різних культур виникає нова транснаціональна культура пам'яті, в якій дивергентні тлумачення зіштовхуються, події оцінюються по-новому, а наративи, які передаються від покоління до покоління, трансформуються.

Водночас зауважено, що спостерігається подальша еволюція транснаціональної літератури як міжкатегоріального поняття. Викладені думки, спостереження та висновки - це лише пошуки у вивченні складних i різноаспектних питань літературознавства.

Ключові слова: транснаціональна література, трансмігрант, транснаціональна культура пам’яті.

Halytska Olena. From the Literature of Guest Workers to the Transnational Literature: the Main Issues and the Research Aspect. The transnational literature is of particular importance in the modern German space. It assumes a transcultural character, which is realized through multilingual or mixed speech tools. In the modern cultural polylogue, there is no complete synthesis and blending, which causes constant logical changes in the understanding and interpretation of the transnational literature concept. The reservation and representation of cultural identity in the works of the German transnational authors of the XXI century enable to model a new cultural cognition in the country, based on the ideas of multiculturalism, ethnic pluralism. This promotes the assimilation of migrants, but not absolute, but with the preservation of their cultural identity. The transnational authors are fighting for their existence in the literary space of Germany. 
The article draws attention to the significant number of terms denoting the concept of the transnational literature. Their terminological delimitation or assimilation is carried out. Based on the study of lexicographic materials and modern linguistic, cultural and literary works, numerous terminology is analyzed: the intercultural literature, the Shamisso literature, the migrant literature, the guest workers' literature, the minority literature, the foreigners' literature, the immigrants' literature, the migration literature, the lost literature, etc. The first works by the transnational authors were devoted to overcoming conflicts caused by prejudice against guest workers. This issue has been replaced by the search for oneself and one's place in the new transcultural space, the search for a third space for new hybrid transnational identities in the zone of blurring and interference. In other words, crossing the borders of different cultures creates a new transnational culture of memory, in which divergent interpretations collide, events are re-evaluated, and narratives, that are passed down from generation to generation, are transformed. However, it is pointed out that there is a further evolution of the transnational literature as an intercategorical concept. The stated ideas, observations, and conclusions are only searches in the study of complex and multifaceted literary issues.

Key words: transnational literature, transmigrant, transnational culture of memory.

DOI: https://doi.org/10.32782/2410-0927-2020-12-8

УДК 811.133 .1 '373.46:53

Оксана Галян

\section{МЕТОДОЛОГІЯ НАУКОВИХ ДОСЛІДЖЕНЬ ФІЗИЧНОЇ ТЕРМІНОСИСТЕМИ ФРАНЦУЗЬКОЇ МОВИ}

У роботі розглянуто послідовність проведення наукової розвідки щодо аналізу теоретичної та емпіричної інформації для пізнання спеціальних понять у фізичній терміносистемі. Виділено два рівні методологічного аналізу: загальнонауковий, пов'язаний із аналізом методів, принципів, форм знань із різних наукових галузей у відповідності до об'єкта і предмета дослідження та конкретно-науковий, зумовлений обгрунтуванням принципів, норм та методів в конкретній науковій діяльності.

Для характеристики елементів фізичної терміносистеми необхідно застосувати описовий та структурний методи, а також систематизацію отриманої інформації. Встановлено, що аналіз складних фізичних термінів необхідно здійснювати поєднанням компонентного аналізу та зіставного методу. Це дозволить виділити терміноелементи і зіставити їх значення з лексемами, які мають спільну семантику. За допомогою семного аналізу встановлено семантичну структуру термінологічних одиниць, виділено смислові елементи (семи) та прокласифіковано за родовою/видовою ознаками. Метод моделювання використано для аналізу багатокомпонентних терміносполук, а з допомогою індуктивного аналізу виділено родові та видові наукові поняття.

Смисл наукового фізичного поняття розкрито за допомогою дефініцій, але поле його поширення визначено ознаками, відповідно до яких відбувається класифікація термінів. Унаслідок класифікаційного поділу угворено підкласи із предметів, явищ, процесів, властивостей і т. ін., що охоплює загальне наукове поняття.

Для дослідження термінів-словосполучень у терміносистемах проведено морфологічний аналіз їхніх компонентів та встановлено взаємозв'язок між ними. Порівняння таких даних із дослідженнями інших терміносистем та словосполучень із загальновживаною лексикою дозволило спрогнозувати ймовірні тенденції розвитку сучасної терміносистеми французької мови.

Методика дослідження скорочених словоформ полягає у виявленні закономірностей, принципів утворення абревіатур та функціонування у фаховому тексті. Встановлення повної вихідної форми лексичних словоформ $\epsilon$ недостатнім для розуміння смислу абревіатури, оскільки значення останньої в конкретному контексті може дещо змінюватись.

Ключові слова: метод дослідження, фізичний термін, семантика, наукове поняття, лексико-семантичні відношення.

Вступ. Створення та побудова наукової терміносистеми, формування методологічних основ та впорядкування термінів - це основні завдання сучасної французької лінгвістики. Методологію дослідження терміносистеми викладено в працях вітчизняних та зарубіжних мовознавців, а саме: Г. О. Винокура [17], Б. М. Головіна [7], В. П. Даниленко [8], Т. Р. Кияка [9], М. П. Кочергана [10]. В. М. Лейчика [11], Д. С. Лотте [12; 13], А. В. Суперанської [15]. Важливість правильного вибору методів і засобів дослідження пов'язана з тим, що (незалежно від того,чи термін уводиться для представлення нового поняття, чи з метою заміни наявного) потрібно здійснити аналіз та наукове обгрунтування необхідності функціонування нової лексичної одиниці в цілісній мовній системі.

(C) Галян О., 2020 